\title{
Administration of an Antioxidant Prevents Lymphoma Development in Transmitochondrial Mice Overproducing Reactive Oxygen Species
}

\author{
Haruka YAMANASHI ${ }^{1 \dagger}$, Osamu HASHIZUME ${ }^{2)}$, Hiromichi YONEKAWA ${ }^{3)}$, \\ Kazuto NAKADA ${ }^{2,4,5)}$, and Jun-Ichi HAYASHI ${ }^{2,4,5)}$ \\ 1) Graduate School of Life and Environmental Sciences, University of Tsukuba, 1-1-1 Tennodai, Tsukuba, Ibaraki \\ 305-8572, Japan \\ ${ }^{2)}$ Faculty of Life and Environmental Sciences, University of Tsukuba, 1-1-1 Tennodai, Tsukuba, Ibaraki 305-8572, \\ Japan \\ ${ }^{3)}$ Center for Basic Technology Research, The Tokyo Metropolitan Institute of Medical Science, 2-1-6 Kamikitazawa, \\ Setagaya-ku, Tokyo, 156-8506, Japan \\ 4) International Institute for Integrative Sleep Medicine (WPI-IIIS), University of Tsukuba, 1-1-1 Tennodai, Tsukuba, \\ Ibaraki 305-8575, Japan \\ ${ }^{5)}$ Life Science Center of Tsukuba Advanced Research Alliance, University of Tsukuba, 1-1-1 Tennodai, Tsukuba, \\ Ibaraki 305-8577, Japan
}

\begin{abstract}
Because of the difficulty to exclude possible involvement of nuclear DNA mutations, it has been a controversial issue whether pathogenic mutations in mitochondrial DNA (mtDNA) and the resultant respiration defects are involved in tumor development. To address this issue, our previous study generated transmitochondrial mice (mito-mice-ND6 ${ }^{13997}$ ), which possess the nuclear and mtDNA backgrounds derived from C57BL/6J (B6) strain mice except that they carry B6 mtDNA with a G13997A mutation in the $m t-N d 6$ gene. Because aged mito-mice-ND6 ${ }^{13997}$ simultaneously showed overproduction of reactive oxygen species (ROS) in bone marrow cells and high frequency of lymphoma development, current study examined the effects of administrating a ROS scavenger on the frequency of lymphoma development. We used $\mathrm{N}$-acetylcysteine (NAC) as a ROS scavenger, and showed that NAC administration prevented lymphoma development. Moreover, its administration induced longevity in mito-mice-ND6 ${ }^{13997}$. The gene expression profiles in bone marrow cells indicated the upregulation of the Fas/ gene, which can be suppressed by NAC administration. Given that natural-killer (NK) cells mediate the apoptosis of various tumor cells via enhanced expression of genes encoding apoptotic ligands including Fas/ gene, its overexpression would reflect the frequent lymphoma development in bone marrow cells. These observations suggest that continuous administration of an antioxidant would be an effective therapeutics to prevent lymphoma development enhanced by ROS overproduction. Key words: antioxidant, lymphoma prevention, mouse mtDNA mutation, ROS overproduction
\end{abstract}

\section{Introduction}

Mitochondrial DNA (mtDNA) mutations that induce mitochondrial respiration defects have been proposed to be involved in aging and age-associated disorders including tumor development [13, 14, 18, 23, 24]. Moreover,

(Received 1 May 2014 / Accepted 27 May 2014 / Published online in J-STAGE 22 July 2014)

Address corresponding: J. Hayashi, Faculty of Life and Environmental Sciences, University of Tsukuba, 1-1-1 Tennodai, Tsukuba, Ibaraki 305-8572, Japan

${ }^{+}$These two authors contributed equally to this study.

(C)2014 Japanese Association for Laboratory Animal Science 
mitochondrial respiration defects caused by nuclear DNA mutations and the resultant enhanced glycolysis under normoxic conditions, i.e. the Warburg effect, are reported to be responsible for tumor development $[4,7,16,19]$. Therefore, it is also possible that mitochondrial respiration defects caused by an age-associated accumulation of mtDNA mutations induce the Warburg effect via compensatory upregulation of aerobic glycolysis, resulting in tumor development. In fact, somatic mutations were preferentially accumulated in tumor mtDNA [6, 10, 21]. However, there has been no direct evidence for the involvement of mtDNA mutations in the Warburg effect and in tumor development, because of the difficulty to exclude possible involvement of nuclear DNA mutations in these processes [2].

Our previous studies $[1,11,12]$ resolved this issue by the use of intercellular mtDNA transfer technology between mouse cells expressing different phenotypes related to tumors. While mtDNA introduced from tumor cells into normal cells did not induce tumorigenicity [1], mtDNA exchange between poorly and highly metastatic lung carcinoma cells provided convincing evidence that a somatic G13997A mtDNA mutation in the $m t-N d 6$ gene, which encodes subunit 6 of respiration complex I (NADH dehydrogenase), reversibly controls development of highly metastatic potentials [11]. In this case, the induction of high metastasis was not due to the Warburg effect, but to overproduction of the reactive oxygen species (ROS) [12]. Subsequently, G13997A mtDNA was transferred from highly metastatic carcinoma cells into mouse female germ line [26] to examine the effect of the G13997A mtDNA on tumor-related phenotypes. The resultant transmitochondrial mice possessing only G13997A mtDNA, named mito-mice-ND6 ${ }^{13997}$, showed high frequently of lymphoma formation with aging [9], providing convincing evidence for the involvement of the mtDNA mutations in tumor development.

Based on these observations, this study examined whether continuous administration of an antioxidant prevents lymphoma development, and corresponds to an effective therapeutics to protect lymphoma development in mito-mice-ND6 ${ }^{13997}$. We also examined the mechanisms of how ROS induce lymphoma formation in mitomice-ND6 ${ }^{13997}$.

\section{Materials and Methods}

\section{Ethical statement}

All animal experiments were performed in accordance with protocols approved by the experimental animal committee of the University of Tsukuba, Japan.

\section{Mice}

Old inbred C57BL/6J Jcl (B6) mice were obtained from CLEA Japan. Mito-mice-ND6 ${ }^{13997}$ were generated in our previous work [26]. We maintained B6 mice and mito-mice-ND6 ${ }^{13997}$ sharing a common nuclear DNA background by repeated backcrossing of their females with B6 males. Animals were housed in groups of up to 5 in individually ventilated cages under standard conditions $\left(22^{\circ} \mathrm{C}, 12 \mathrm{~h}\right.$ light-dark cycle) receiving food and water ad libitum. Male mice were used in the experiments, and were monitored everyday for general health and signs of tumor burden such as hunched postures, ruffled coats and respiratory distress were humanely killed. Moribund mice were euthanized by cervical dislocation under general anesthesia (Avertin, 1.25\%, $0.2 \mathrm{ml} / 20 \mathrm{~g}$ body weight, intraperitoneally).

\section{NAC administration}

At 15 months old, sixteen mito-mice-ND6 ${ }^{13997}$ were divided at random into two groups. One group was given drinking water containing $10 \mathrm{~g} / 1$ of NAC (SIG$\mathrm{MA}$ ), and another group was given regular water. NAC supplemented water and regular water were prepared fresh everyday.

\section{Histological analyses}

Formalin-fixed, paraffin-embedded serial sections $(10 \mu \mathrm{m})$ were used for histological analyses. Hematoxylin-and-eosin-stained sections were used for histopathological analysis to identify tumor tissues. The immunohistological analysis was performed with antibody to CD45 to determine whether the tumor tissues originated from leukocytes, and subsequently with antibodies to B220 and CD3 to determine whether the tumor tissues were of B-cell or T-cell origin, respectively. Deparaffinized slides were boiled for antigen retrieval, then incubated with rat anti-mouse CD45 (clone 30-F11; BD Biosciences, Cat. No. 550539) at a dilution of 1:200 or rat anti-mouse B220 (clone RA3-6B2; BD Biosciences, Cat. No. 553085) at a dilution of 1:50 or goat anti-mouse CD3 (clone M-20; Santa Cruz, Cat. No. sc-1127) at a 
dilution of 1:50. Biotin-conjugated goat anti-rat $\operatorname{IgG}(\mathrm{BD}$ Biosciences, Cat. No. 559286) and rabbit anti-goat IgG (Vector Laboratories, Cat. No. BA-5000) were used as secondary antibody at a dilution of 1:50 and 1:500, respectively. Detection of CD45, B220 and CD3 were performed using avidin-biotin complex methodologies (Vectastain Elite ABC Kit, Vector Laboratories) with DAB staining (Anti-Ig HRP Detection Kit, BD Pharmingen). Sections were counterstained with hematoxylin.

\section{PCR array analysis}

Mice were euthanized after 4-week administration of $\mathrm{NAC}$ and bone marrow cells were isolated from mice thighbones and shinbones. Total RNA was extracted by ISOGEN (Nippon Gene) from mouse bone marrow cells. RNA samples were subjected to DNase I treatment (Invitrogen) to eliminate DNA contaminants and reverse transcribed using Oligo (dT) $)_{12-18}$ primer, $10 \mathrm{mM}$ dNTP Mix, 0.1 M DTT, RNase Out Recombinant Ribonuclease Inhibitor, and SuperScript II-Reverse Transcriptase (Invitrogen). cDNA samples were subjected to RNase $\mathrm{H}$ treatment (Invitrogen), and applied according to the manufacturer's protocol to a $\mathrm{RT}^{2}$ profiler $\mathrm{PCR}$ array real-time PCR reaction. Real-time monitoring PCR was performed with SYBR Green PCR Master Mix (QIAGEN) and an ABI PRISM 7900HT sequence detection system (Applied Biosystems). Mouse Cancer Pathway Finder RT ${ }^{2}$ Profiler PCR Array PAMM-033Z (SABiosciences) was performed to profile the expression of 84 genes related to the cancer pathway $(n=3)$. The expression profiling was performed using $\Delta \Delta \mathrm{Ct}$ methods according to manufacturer's protocols. The relative expression level for each gene was represented as cycle threshold (Ct). Normalized expression level was calculated as $\Delta \mathrm{Ct}=\mathrm{Ct}$ (Gene of interest) $-\mathrm{Ct}$ (control). The average $\Delta \mathrm{Ct}$ from three mice was calculated as the relative expression level of each gene. Differential expression was calculated as $\Delta \Delta \mathrm{Ct}=\mathrm{Ave} \Delta \mathrm{Ct}$ (sample mice) Ave $\Delta \mathrm{Ct}$ (control mice). Fold change was calculated as $2^{-\Delta \Delta \mathrm{Ct}}$. The $P$ values are calculated based on a Student $t$-test of the replicate $2^{-\Delta \mathrm{Ct}}$ values for each gene in the control group and treatment groups.

\section{Statistical analysis}

We analyzed data with the Student's $t$-test. KaplanMeier curves were assessed with the log-rank test. Values with $P<0.05$ were considered significant.

\section{Results}

This study examined the idea that the frequent lymphoma development in the aged male mito-miceND $6^{13997}$ could be prevented by the administration of an antioxidant, if ROS overproduction is responsible for the lymphoma development. Since most mito-miceND6 ${ }^{13997}$ began to develop lymphoma more than 18 months after the birth [9], we used 15-month-old males for continuous administration of $N$-acetylcysteine (NAC) as a ROS scavenger. Eight of 16 mito-mice-ND6 ${ }^{13997}$ were treated with NAC from 15 months after the birth by oral administration based on the procedure reported previously [5]. The remaining eight mito-mice-ND6 ${ }^{13997}$ were not treated with NAC. Seven 15-month-old B6 males untreated with NAC were used as controls.

First, we examined whether the NAC treatment would affect the frequency of lymphoma development and the lifespan. Median survival times of NAC-treated mitomice-ND6 ${ }^{13997}$, untreated mito-mice-ND6 ${ }^{13997}$ and untreated B6 mice were 29.3, 22.5 and 25.8 months, respectively (Figs. 1A and B). No statistical significance of median survival times was observed between untreated mito-mice-ND6 $6^{13997}$ and untreated B6 mice (Fig.1A). These results are consistent with our previous observations [9]. In contrast, NAC-treated mito-miceND6 ${ }^{13997}$ showed longer survival times than untreated mito-mice-ND6 ${ }^{13997}$ with statistical significance (Fig. 1B), while no statistical significance was present between NAC-treated mito-mice-ND6 ${ }^{13997}$ and untreated B6 mice (Figs. 1A and B). The median survival times of untreated mito-mice-ND6 $6^{13997}$ with and without lymphoma-like abnormalities were 24.5 and 20.5 , respectively, and were not different with statistical significance (Fig. 1C).

Gross necropsy of all dead or euthanized moribund mice showed that three of eight mito-mice-ND6 ${ }^{13997}(38 \%)$ and one of seven B6 mice (14\%) had macroscopic lymphoma-like abnormalities, including enlarged spleen, liver, and nodular tumors, but none of eight NAC-treated mito-mice-ND6 ${ }^{13997}$ had these abnormalities (Table 1). Histological analyses of abnormal tissues revealed that all were hematopoietic neoplasms and positive for the pan-leukocyte marker CD45 (Table 1 and Fig. 2 ). These observations suggest that these hematological neoplasms may correspond to lymphoma cells, although current study did not examine copy-number variations (CNVs) in nuclear genomes to show chromosomal instability [9]. 
A

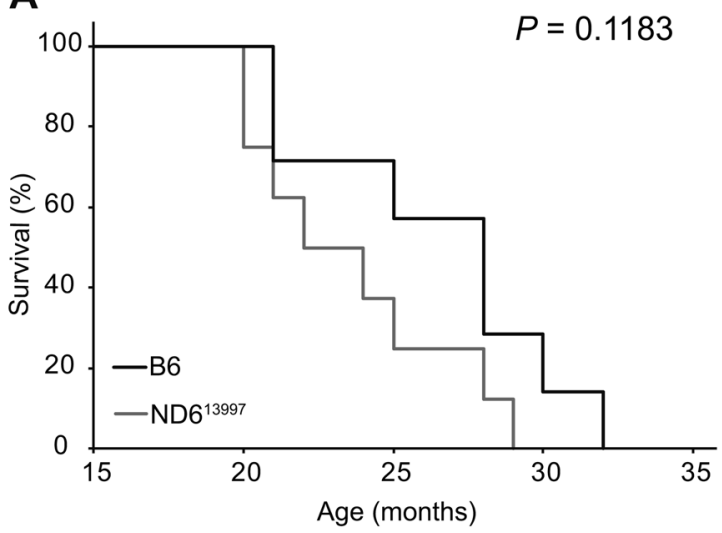

C

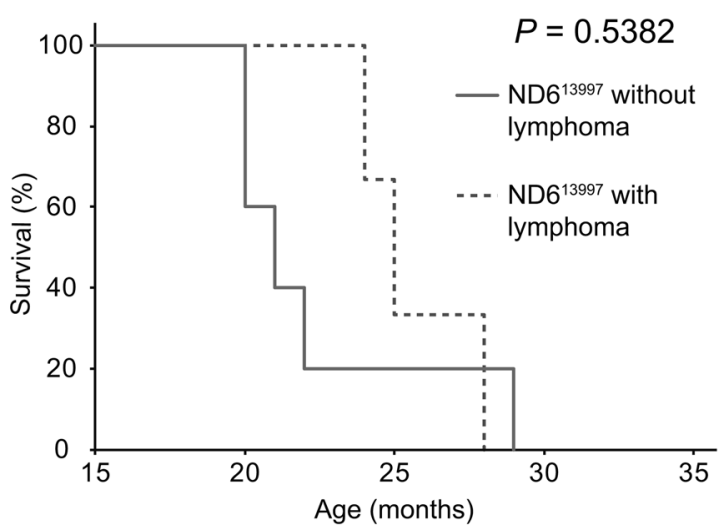

B

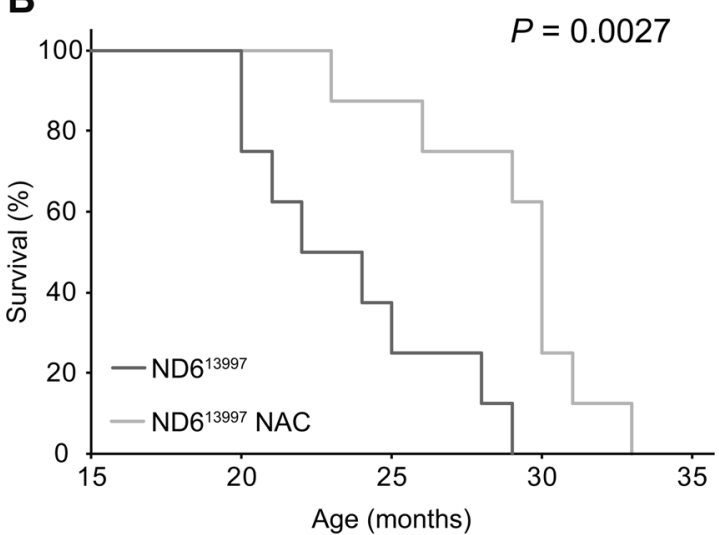

Fig. 1. Kaplan-Meier survival curves of mito-miceND6 ${ }^{13997}$. (A) Comparison of lifespans between B6 mice and mitomiceND6 ${ }^{13997}$. Median survival times of B6 mice $(n=7)$ and mito-miceND6 ${ }^{13997}(n=8)$ were 25.8 and 22.0 months, respectively. (B) Comparison of lifespans of between NAC-treated and untreated mito-miceND6 ${ }^{13997}$. NAC administration was started from 15 months after the birth to the end of their lives. Median survival times of mitomiceND $6^{13997}(\mathrm{n}=8)$ and mito-miceND6 ${ }^{13997}$ treated with NAC $(\mathrm{n}=8)$ were 22.0 and 29.3 months, respectively. (C) Comparison of lifespans between mito-miceND6 ${ }^{13997}$ that died with and without detectable lymphoma. Median survival times of mito-miceND6 ${ }^{13997}$ with detectable lymphoma $(n=3)$ and mito-miceND6 ${ }^{13997}$ without detectable lymphoma $(\mathrm{n}=5)$ were 24.5 and 20.5 months, respectively.

Table 1. Frequencies of lymphoma development in mice

\begin{tabular}{|c|c|c|c|c|c|c|c|c|c|}
\hline \multirow{2}{*}{ Mouse strains } & \multirow{2}{*}{ No. of mice } & \multirow{2}{*}{$\begin{array}{l}\text { No. of mice } \\
\text { with tumor }\end{array}$} & \multicolumn{3}{|c|}{ Tissues with tumor } & \multicolumn{3}{|c|}{ Histological analysis } & \multirow{2}{*}{ Cell lineage } \\
\hline & & & Spleen & Liver & Lymph node & CD45 & B220 & $\mathrm{CD} 3$ & \\
\hline B6 & 7 & 1 & + & + & + & + & + & - & B-cell \\
\hline \multirow[t]{3}{*}{ Mito-mice-ND6 ${ }^{13997}$} & 8 & 3 & + & - & + & + & + & - & B-cell \\
\hline & & & + & - & - & + & - & + & T-cell \\
\hline & & & + & + & + & + & + & - & B-cell \\
\hline $\begin{array}{l}\text { Mito-mice-ND6 }{ }^{13997} \\
\text { (NAC-treated) }\end{array}$ & 8 & 0 & & & & & & & \\
\hline
\end{tabular}

Three tumors were of B-cell origin, expressing the B-cell maker B220, while one mito-mouse-ND6 ${ }^{13997}$ developed T-cell lymphoma staining positive for the T-cell marker CD3 (Table 1). These data indicate that NAC administra- tion is an effective therapeutics to prevent lymphoma development caused by ROS overproduction. However, further works using more numbers of animals than those used in this study are required to confirm this idea. 


\section{A}

\section{Liver}

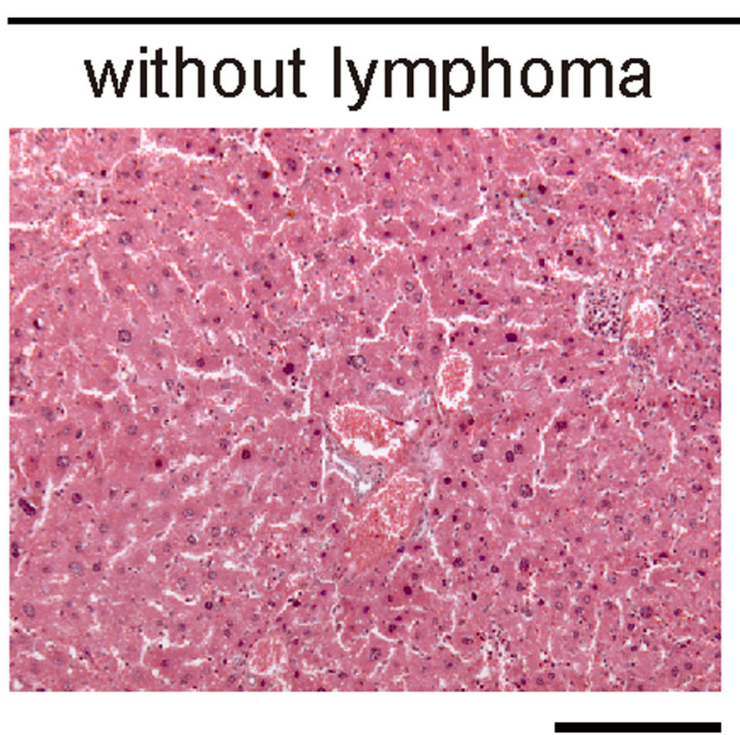

\section{with lymphoma}

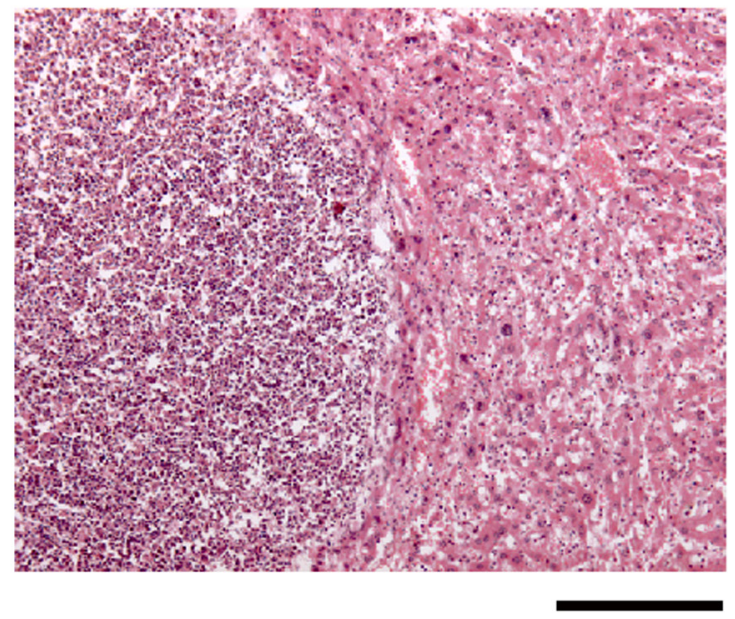

\section{B}

\section{Liver with lymphoma}
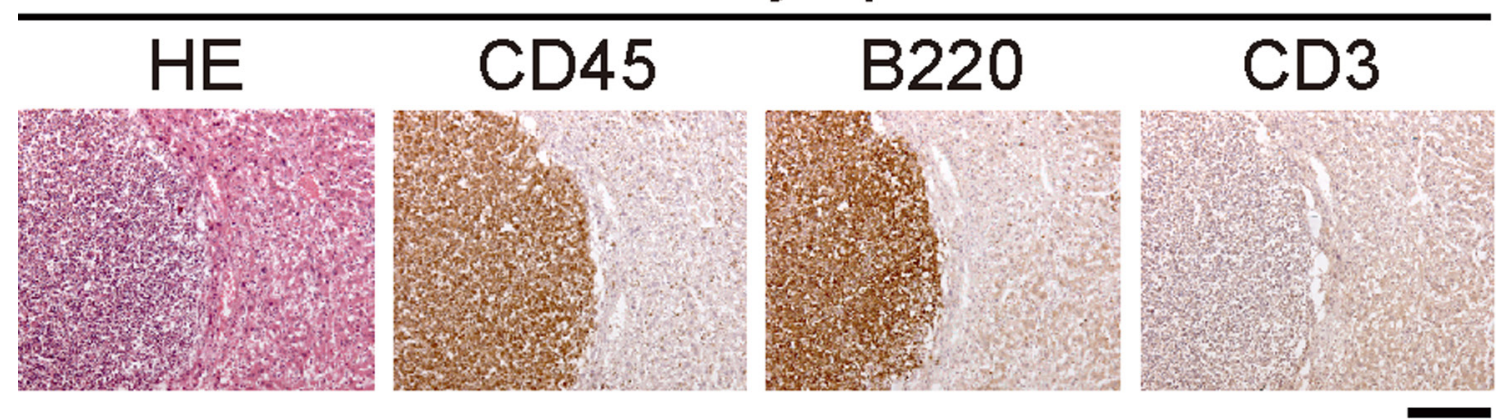

Fig. 2. Histological analyses for identification of lymphoma cells in the liver from mito-miceND6 ${ }^{13997}$. (A) Hematoxylin and eosin (HE) staining of the liver sections from mito-miceND6 ${ }^{13997}$. HE staining of the liver sections was carried out to identify tumor-like abnormalities in the liver. Left and right panels represent normal liver and liver with tumorlike abnormalities, respectively. (B) Histological analysis of serial sections of the liver with tumor-like abnormalities. HE, hematoxylin-eosin staining to show tumor formation; CD45, immunohistochemistry using antibody to CD45 to detect leukocytes; B220, immunohistochemistry using antibody to B220 to detect B cells; CD3, immunohistochemistry using antibody to CD3 to detect T cells. Because this tissue was stained positively with CD45 and B220, but not with CD3, the results represent abnormal growth of B cell-lymphoma, but not T cell-lymphoma, in the liver. (Scale bars, $200 \mu \mathrm{m}$ ).

Then, we examined the mechanism of frequent lymphoma development, which can be prevented by administration of an antioxidant to mito-mouse-ND6 ${ }^{13997}$. Our previous study showed that no tumors other than lymphoma were developed, and that ROS were overproduced in bone marrow cells but not in splenocytes [9]. It is therefore likely that the ROS overproduction in the bone marrow cells of mito-mouse-ND6 ${ }^{13997}$ is crucial for lymphoma development. Thus, we used bone marrow cells to identify genes that would be responsible for frequent lymphoma development in mito-mice-ND6 ${ }^{13997}$, and compared the expression of the genes related to cancer pathway (transformation and tumorigenesis) between mito-mice-ND6 ${ }^{13997}$ and B6 mice using PCR array (Mouse Cancer Pathway Finder RT ${ }^{2}$ Profiler PCR Array). Because most mito-mice-ND6 ${ }^{13997}$ began to develop 

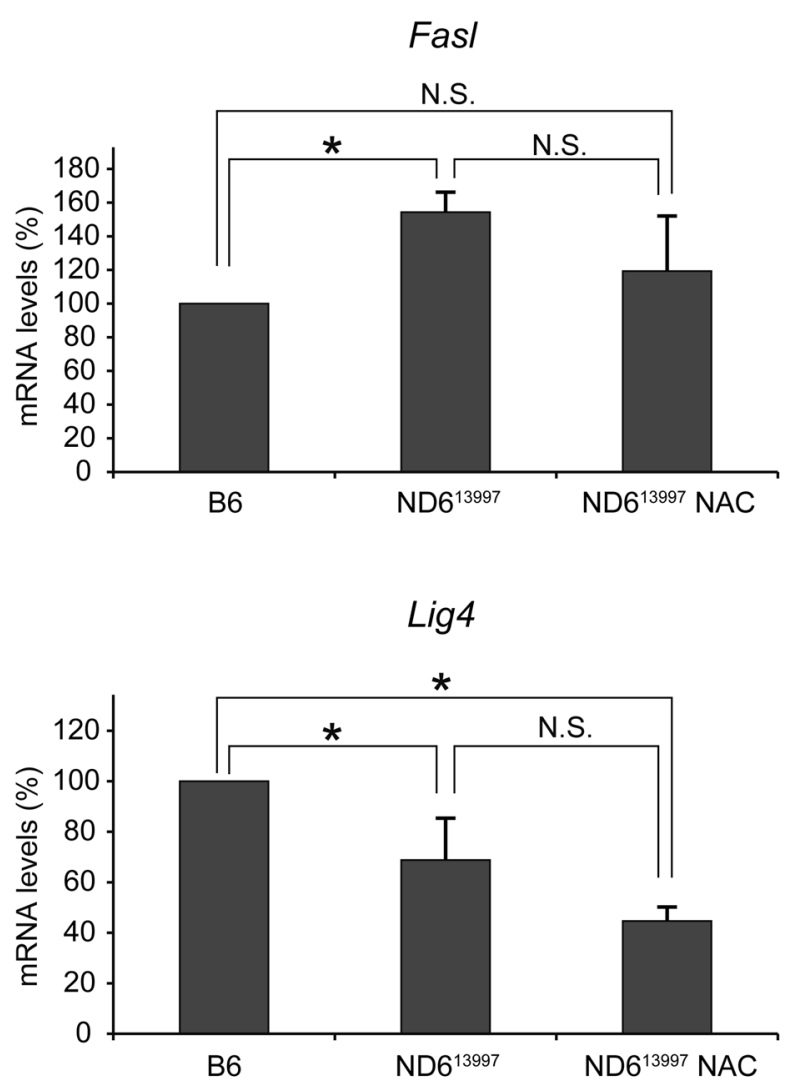

Fig. 3. Cancer related gene expression profiles of bone marrow cells. RT ${ }^{2}$ Profiler mouse cancer pathway finder PCR array was performed on bone marrow cells. Two genes of 84 cancer pathway-related genes, Fasl and Lig4, were differentially expressed between bone marrow cells of B6 mice and mito-mice-ND6 ${ }^{13997}$. However, only the Fasl gene expression was reversibly regulated to normal levels by administration of NAC. Values were normalized to a pool of housekeeping genes on the array by $\Delta \Delta \mathrm{Ct}$ and are reported as the fold change in gene expression relative to healthy B6 bone marrow cells. Data are represented as mean values with $\mathrm{SD}(\mathrm{n}=3)$. *, $P<0.05$.

lymphoma 18 months after the birth, we used 15-monthold male mice for this experiment to exclude the influence of the developed lymphoma cells.

Out of 84 cancer pathway-related genes represented on Mouse Cancer Pathway Finder RT ${ }^{2}$ Profiler PCR Array (Supplemental table 1), only two genes, Fasl and Lig4 related to apoptosis and DNA repair, respectively, were selected as genes being differentially expressed between bone marrow cells of B6 and mito-miceND6 ${ }^{13997}$ (Fig. 3). Then, we examined whether expression levels of these genes can be changed to the levels of B6 mice by administration of NAC for 4 weeks to mitomice-ND6 ${ }^{13997}$. The results showed that Fasl overexpres- sion in mito-mice-ND6 ${ }^{13997}$ was suppressed to B6 levels by NAC administration, while suppressed expression of Lig4 levels did not restore to B6 levels (Fig. 3). These results indicated the association of $\mathrm{Fasl}$ overexpression to frequent development of lymphoma in mito-miceND6 ${ }^{13997}$.

\section{Discussion}

This study addressed two important issues of whether treatment of the mito-mice-ND6 ${ }^{13997}$ with an antioxidant could be an effective therapeutics to prevent lymphoma development mito-mice-ND6 ${ }^{13997}$, and how ROS overproduction caused by G13997A mtDNA introduced from highly metastatic lung carcinoma cells induces lymphoma development in mito-mice-ND6 $6^{13997}$.

Concerning the first issue on the therapeutics of lymphoma development, the results in this study showed that administration of NAC, one of the frequently used antioxidants, is very effective to prevent lymphoma development in mito-mice-ND6 ${ }^{13997}$. Our previous study proposed the idea that ROS overproduction, but not lactate overproduction, is responsible for frequent development of lymphoma in mito-mice-ND6 $6^{13997}$ with G13997A mtDNA based on the observations that lymphoma was not developed in different mito-mice-COI ${ }^{6589}$ that showed lactate overproduction but did not show ROS overproduction [9]. If this idea is correct, it can be predicted that administration of the antioxidants would prevent lymphoma development in mito-mice-ND6 ${ }^{13997}$. Current study examined this prediction and showed that NAC administration is effective to prevent lymphoma development caused by ROS in mito-mice-ND6 ${ }^{13997}$. However, further works are required to show that NAC administration prevents ROS overproduction in bone marrow cells of mito-mice-ND6 ${ }^{13997}$.

While NAC administration prevents lymphoma development (Table 1), it also induced longevity in mitomice-ND6 13997 (Fig. 1B). Median survival times of B6 mice, mito-mice-ND6 ${ }^{13997}$, and NAC-treated mito-miceND6 ${ }^{13997}$ were 25.8, 22.0, and 29.3, respectively (Figs. 1A and B). The longer median survival times of NAC-treated mito-mice-ND6 ${ }^{13997}$ would not be due to the prevention of lymphoma formation by NAC administration, because the median survival times of untreated mito-mice-ND6 ${ }^{13997}$ with and without lymphoma were 24.5 and 20.5, respectively, and were not different with statistical significance (Fig. 1C). Since it appears to be 
controversial whether oxidative stress suppresses longevity or not $[20,25]$, we could not at present explain why NAC administration induced longevity in mitomice-ND6 ${ }^{13997}$. To examine this issue, we have to carry out NAC administration not only to mito-mice-ND6 ${ }^{13997}$ but also to B6 mice using more numbers of animals.

Concerning the second issue of how ROS overproduction caused by G13997A mtDNA induced lymphoma development, this study showed that overexpression of Fasl is related the process, because its overexpression in mito-mice-ND6 ${ }^{13997}$ was suppressed to normal levels by NAC administration (Fig. 3). Given that naturalkiller (NK) cells mediate the apoptosis of various tumor cells via the expression of genes encoding tumor necrosis factor-family ligands including the Fasl gene [22], it would not be conceivable that its overexpression is responsible for lymphoma development. Probably, overexpression of the Fasl gene in NK cells simply reflects the progress of the frequent lymphoma development in bone marrow cells of mito-mice-ND6 ${ }^{13997}$. Therefore, we could not at present explain the mechanisms of how ROS overproduction induces frequent lymphoma development in aged mito-mice-ND6 ${ }^{13997}$.

Then, a question is why mito-mice-ND6 ${ }^{13997}$ preferentially develop lymphoma [9] (Table 1), even though ROS induces various types of cellular damages, leading to genomic instability that can result in the development of various types of tumors [15]. One answer to this question would be that nuclear genetic background of B6 strain we used for generation of mito-mice-ND6 ${ }^{13997}$ is prone to develop lymphoma $[3,8,17]$. Based on these observations, we would like to propose an idea that G13997A mtDNA alone would not induce lymphoma development in the absence of B6 nuclear genetic background. To examine this idea, we are going to generate new mito-mice-ND6 ${ }^{13997}$ carrying G13997A mtDNA but carrying different nuclear genetic background derived from different mouse strains that are not prone to develop lymphoma by backcrossing female mito-miceND6 ${ }^{13997}$ to males from different mouse strains.

\section{Acknowledgments}

This work was supported by Grants-in-Aid for Scientific Research A 25250011 (to J.-I.H.), Scientific Research A 23240058 (to K.N.), and Scientific Research on Innovative Areas 24117503 (to J.-I.H.) from the Japan Society for the Promotion of Science. This work was supported also by the World Premier International Research Center Initiative; Ministry of Education, Culture, Sports, Science and Technology-Japan (to K.N. and J.-I.H.).

\section{References}

1. Akimoto, M., Niikura, M., Ichikawa, M., Yonekawa, H., Nakada, K., Honma, Y., and Hayashi, J. 2005. Nuclear DNA but not mtDNA controls tumor phenotypes in mouse cells. Biochem. Biophys. Res. Commun. 327: 1028-1035. [Medline] [CrossRef]

2. Augenlicht, L.H. and Heerdt, B.G. 2001. Mitochondria: integrators in tumorigenesis? Nat. Genet. 28: 104-105. [Medline] [CrossRef]

3. Balmain, A. and Nagase, H. 1998. Cancer resistance genes in mice: models for the study of tumour modifiers. Trends Genet. 14: 139-144. [Medline] [CrossRef]

4. Baysal, B.E., Ferrell, R.E., Willett-Brozick, J.E., Lawrence, E.C., Myssiorek, D., Bosch, A., van der Mey, A., Taschner, P.E., Rubinstein, W.S., Myers, E.N., Richard, C.W. 3rd., Cornelisse, C.J., Devilee, P., and Devlin, B. 2000. Mutations in SDHD, a mitochondrial complex II gene, in hereditary paraganglioma. Science 287: 848-851. [Medline] [CrossRef]

5. De Flora, S., D’Agostini, F., Masiello, L., Giunciuglio, D., and Albini, A. 1996. Synergism between N-acetylcysteine and doxorubicin in the prevention of tumorigenicity and metastasis in murine models. Int. J. Cancer 67: 842-848. [Medline] [CrossRef]

6. Fliss, M.S., Usadel, H., Caballero, O.L., Wu, L., Buta, M.R., Eleff, S.M., Jen, J., and Sidransky, D. 2000. Facile detection of mitochondrial DNA mutations in tumors and bodily fluids. Science 287: 2017-2019. [Medline] [CrossRef]

7. Gottlieb, E. and Tomlinson, I.P. 2005. Mitochondrial tumour suppressors: a genetic and biochemical update. Nat. Rev. Cancer 5: 857-866. [Medline] [CrossRef]

8. Harvey, M., McArthur, M.J., Montgomery, C.A. Jr., Bradley, A., and Donehower, L.A. 1993. Genetic background alters the spectrum of tumors that develop in p53-deficient mice. FASEB J. 7: 938-943. [Medline]

9. Hashizume, O., Shimizu, A., Yokota, M., Sugiyama, A., Nakada, K., Miyoshi, H., Itami, M., Ohira, M., Nagase, H., Takenaga, K., and Hayashi, J. 2012. Specific mitochondrial DNA mutation in mice regulates diabetes and lymphoma development. Proc. Natl. Acad. Sci. USA 109: 10528-10533. [Medline] [CrossRef]

10. He, Y., Wu, J., Dressman, D.C., Iacobuzio-Donahue, C., Markowitz, S.D., Velculescu, V.E., Diaz, L.A. Jr., Kinzler, K.W., Vogelstein, B., and Papadopoulos, N. 2010. Heteroplasmic mitochondrial DNA mutations in normal and tumour cells. Nature 464: 610-614. [Medline] [CrossRef]

11. Ishikawa, K., Takenaga, K., Akimoto, M., Koshikawa, N., Yamaguchi, A., Imanishi, H., Nakada, K., Honma, Y., and Hayashi, J. 2008. ROS-generating mitochondrial DNA mutations can regulate tumor cell metastasis. Science 320: 661664. [Medline] [CrossRef]

12. Ishikawa, K., Hashizume, O., Koshikawa, N., Fukuda, S., 
Nakada, K., Takenaga, K., and Hayashi, J. 2008. Enhanced glycolysis induced by mtDNA mutations does not regulate metastasis. FEBS Lett. 582: 3525-3530. [Medline] [CrossRef]

13. Jacobs, H.T. 2003. The mitochondrial theory of aging: dead or alive? Aging Cell 2: 11-17. [Medline] [CrossRef]

14. Khrapko, K. and Vijg, J. 2009. Mitochondrial DNA mutations and aging: devils in the details? Trends Genet. 25: 91-98. [Medline] [CrossRef]

15. Klaunig, J.E., Kamendulis, L.M., and Hocevar, B.A. 2010. Oxidative stress and oxidative damage in carcinogenesis. Toxicol. Pathol. 38: 96-109. [Medline] [CrossRef]

16. Koppenol, W.H., Bounds, P.L., and Dang, C.V. 2011. Otto Warburg's contributions to current concepts of cancer metabolism. Nat. Rev. Cancer 11: 325-337. [Medline] [CrossRef]

17. Krupke, D.M., Begley, D.A., Sundberg, J.P., Bult, C.J., and Eppig, J.T. 2008. The Mouse Tumor Biology database. Nat. Rev. Cancer 8: 459-465. [Medline] [CrossRef]

18. Loeb, L.A., Wallace, D.C., and Martin, G.M. 2005. The mitochondrial theory of aging and its relationship to reactive oxygen species damage and somatic mtDNA mutations. Proc. Natl. Acad. Sci. USA 102: 18769-18770. [Medline] [CrossRef]

19. Niemann, S. and Müller, U. 2000. Mutations in SDHC cause autosomal dominant paraganglioma, type 3. Nat. Genet. 26: 268-270. [Medline] [CrossRef]
20. Pérez, V.I., Bokov, A., Van Remmen, H., Mele, J., Ran, Q., Ikeno, Y., and Richardson, A. 2009. Is the oxidative stress theory of aging dead? Biochim. Biophys. Acta 1790: 10051014. [Medline] [CrossRef]

21. Polyak, K., Li, Y., Zhu, H., Lengauer, C., Willson, J.K., Markowitz, S.D., Trush, M.A., Kinzler, K.W., and Vogelstein, B. 1998. Somatic mutations of the mitochondrial genome in human colorectal tumours. Nat. Genet. 20: 291-293. [Medline] [CrossRef]

22. Smyth, M.J., Hayakawa, Y., Takeda, K., and Yagita, H. 2002. New aspects of natural-killer-cell surveillance and therapy of cancer. Nat. Rev. Cancer 2: 850-861. [Medline] [CrossRef]

23. Taylor, R.W. and Turnbull, D.M. 2005. Mitochondrial DNA mutations in human disease. Nat. Rev. Genet. 6: 389-402. [Medline] [CrossRef]

24. Wallace, D.C. 1999. Mitochondrial diseases in man and mouse. Science 283: 1482-1488. [Medline] [CrossRef]

25. Yang, W. and Hekimi, S. 2010. A mitochondrial superoxide signal triggers increased longevity in Caenorhabditis elegans. PLoS Biol. 8: e1000556. [Medline] [CrossRef]

26. Yokota, M., Shitara, H., Hashizume, O., Ishikawa, K., Nakada, K., Ishii, R., Taya, C., Takenaga, K., Yonekawa, H., and Hayashi, J. 2010. Generation of trans-mitochondrial mitomice by the introduction of a pathogenic G13997A mtDNA from highly metastatic lung carcinoma cells. FEBS Lett. 584: 3943-3948. [Medline] [CrossRef] 